Joint Irish Section and American Society for Nutrition Meeting, 15-17 June 2011, 70th anniversary: 'Vitamins in early development and healthy ageing: impact on infectious and chronic disease,

\title{
Dietary patterns among pregnant women in Ireland
}

\author{
C. McGowan, J. Byrne, J. Walsh and F. M. McAuliffe \\ School of Medicine and Medical Science, University College Dublin, National Maternity Hospital, Holles Street, Dublin 2
}

Pregnancy is the most important stage of a women's life cycle where nutrition is essential for an optimum pregnancy outcome ${ }^{(1)}$. Dietary pattern analysis is becoming a popular method of determining habitual dietary patterns during pregnancy. Cluster analysis is one technique that can be used to identify clusters or groups of subjects with similar dietary characteristics ${ }^{(2)}$. The aim of this study was to describe the main dietary patterns among a cohort of pregnant women living in Ireland using $k$-means cluster analysis.

Two hundred and fifteen pregnant women were recruited from the antenatal clinic at the National Maternity Hospital. All participants were over 18 years of age, with a healthy singleton pregnancy and with adequate English. A 3-d food diary was completed during each trimester of pregnancy where food and beverage intakes were recorded over three consecutive days including one weekend day. Dietary data were entered into NetWISP version 3.0 (Tinuviel software, Llanfechell, Anglesey, UK) and analysed using $k$-means cluster analysis in PASW statistics version 18.0 (SPSS Inc., Chicago, IL, USA). Thirty-three food groups were extracted from the food file in NetWISP and were standardised to $z$-scores prior to running the analyses so that they had equal weights when distances were computed.

Table 1 shows the dietary patterns identified during each trimester of pregnancy and the principle food groups within each pattern. When all trimesters were combined, two major dietary patterns were identified: 'Healthy' and 'Western'.

Table 1. Major dietary patterns during each trimester of pregnancy assessed by $3-\mathrm{d}$ food diaries $(n 215)$

\begin{tabular}{|c|c|c|c|c|c|c|c|}
\hline \multicolumn{3}{|l|}{ Trimester 1} & \multicolumn{2}{|c|}{ Trimester 2} & \multicolumn{3}{|c|}{ Trimester 3} \\
\hline 'High carb' ( $n$ 137) & 'Healthy' ( $n$ 76) & 'Oily fish' (n 2) & 'Healthy' (n 140) & 'Western' $(n$ 75) & 'Western' (n 106) & 'Healthy' ( $n$ 78) & 'Oily fish' (n 31) \\
\hline All breads & Whole grains & Oily fish & Fruit & White bread & Processed meats & Fruit & Oily fish \\
\hline Pasta/rice & Fruit & Eggs & Whole grains & Beverages & White bread & Cereals & Soup \\
\hline
\end{tabular}

个Beverages, high-energy beverages; carb, carbohydrate.

In conclusion, $k$-means cluster analysis is a useful method in determining the main dietary patterns among pregnant women. These data can now be analysed to assess relationships between dietary patterns during pregnancy and future pregnancy outcome.

1. Keen CL, Clegg MS, Hanna LA et al. (2003) J Nutr 133, 1597S-1605S.

2. Quatromoni PA, Copenhafer DL, Demissie S et al. (2002) J Epidemiol Community Health 56, 381-388. 